\title{
Communication \\ A Low-Cost and Efficient Microstrip-Fed Air-Substrate-Integrated Waveguide Slot Array
}

\author{
Linfeng $\mathrm{Li}^{*}$ and Jie-Bang Yan \\ Electrical and Computer Engineering Department, The University of Alabama, Tuscaloosa, AL 35487, USA; \\ jbyan@ua.edu \\ * Correspondence: 1li74@crimson.ua.edu
}

check for

updates

Citation: Li, L.; Yan, J-B. A Low-Cost and Efficient Microstrip-Fed Air-Substrate-Integrated Waveguide Slot Array. Electronics 2021, 10, 338. https://doi.org/10.3390/

electronics 10030338

Academic Editor: Rafal Przesmycki

Received: 5 January 2021

Accepted: 29 January 2021

Published: 1 February 2021

Publisher's Note: MDPI stays neutral with regard to jurisdictional claims in published maps and institutional affiliations.

Copyright: (c) 2021 by the authors. Licensee MDPI, Basel, Switzerland. This article is an open access article distributed under the terms and conditions of the Creative Commons Attribution (CC BY) license (https:/ / creativecommons.org/licenses/by/ $4.0 /)$.

\begin{abstract}
A microstrip-fed air-substrate-integrated waveguide (ASIW) slot array with high efficiency and low cost is presented. The design cuts out the substrate material within SIW, replaces the vias with metallic sidewalls, and uses a simple microstrip line-waveguide transition to feed the slot array. Radiating slots are cut on a 5-mil brass-plate, which covers the top of the substrate cutout to resemble a hollow waveguide structure. This implementation provides a simple and efficient antenna array solution for millimeter-wave (mm-wave) applications. Meanwhile, the fabrication is compatible with the standard printed circuit board (PCB) manufacturing process. To demonstrate the concept, a 4-element ASIW slot array working at the $\mathrm{n} 257$ band for $5 \mathrm{G}$ communications was designed using low-cost Rogers 4350B and FR4 substrate materials. Our simulation result shows 18\% more efficiency than a conventional SIW slot array using the same substrate. The fabricated prototype shows $|\mathrm{S} 11|<-15 \mathrm{~dB}$ over $27-29 \mathrm{GHz}$ and a peak realized gain of $10.1 \mathrm{dBi}$ at 28.6 GHz. The design procedure, prototyping process, and design analysis are discussed in the paper.
\end{abstract}

Keywords: SIW; waveguide; slot array; mm-wave; 5G

\section{Introduction}

With the rapid development of 5G communication systems and mm-wave automotive radars, better quality, low-cost, and low-loss antennas are in high demand. Substrateintegrated waveguide (SIW), which was first introduced by Deslandes and Wu [1], provides a high-performance solution for mm-wave antenna design. Since then, SIW slot arrays have been analyzed and developed at multiple bands for different applications [2-5]. They are compact and easy to fabricate and integrate with other planar circuits. However, the SIW slot arrays suffer from different sources of loss such as conductor loss, dielectric loss, and wave-leakage loss, which become more significant as frequency goes beyond Kband. Building high-efficiency antennas at mm-wave requires expensive low-loss substrate materials and high-precision manufacturing processes. To overcome these limits, the air-filled structure of the traditional rectangular waveguide has been reconsidered and gradually implemented using the current substrate-integrated circuit (SIC) technique. The numerical analysis in Ranjkesh et al. [6] shows that when using Rogers RO4003 (Rogers Corporation, Chandler, Arizona) as the substrate, the attenuation constant of SIW with an air-cut is reduced by more than $1.5 \mathrm{~Np} / \mathrm{m}$ at $29 \mathrm{GHz}$, compared to that of SIW. More recently, an air-filled SIW (AFSIW) [7] and a slot array using this structure [8] were developed. The simulated antenna array has an efficiency of more than $90 \%$, which benefits from the substrate cut-out and the efficient transition from SIW and AFSIW. However, a part of the waveguide is dielectric-filled. Additionally, the gaps between existing metallic vias can still cause wave-leakage and reduce antenna performance at higher mm-wave frequencies. To eliminate the metallic vias, simplify the transition, and further improve the antenna efficiency, we introduce a tapered microstrip-fed air-SIW (ASIW) slot array with copperplated side walls. The radiating slots are cut on a 5-mil brass layer, which covers the top of the substrate cutout. The copper-plated sidewalls along with the top and bottom 
metallic covers form a hollow waveguide with no dielectric material and no vias. This three-layer structure can be easily aligned with alignment holes and fabricated with the current multi-layer PCB manufacturing process. This paper will be arranged as follows. The antenna array design and fabrication process are discussed in Sections 1 and 2. The simulated and measured antenna performance, the effect of surface roughness on antenna efficiency, and a comparison between the proposed ASIW slot array and the SIW slot array using the same substrate material are presented in Section 3. Finally, the conclusion is given in Section 4.

\section{Antenna Array Design}

The 3-dimensional (3D) structure of the proposed $1 \times 4$ slot array is shown in Figure 1a. The printed circuit board (PCB) multilayer stack-up is shown in Figure 1b.

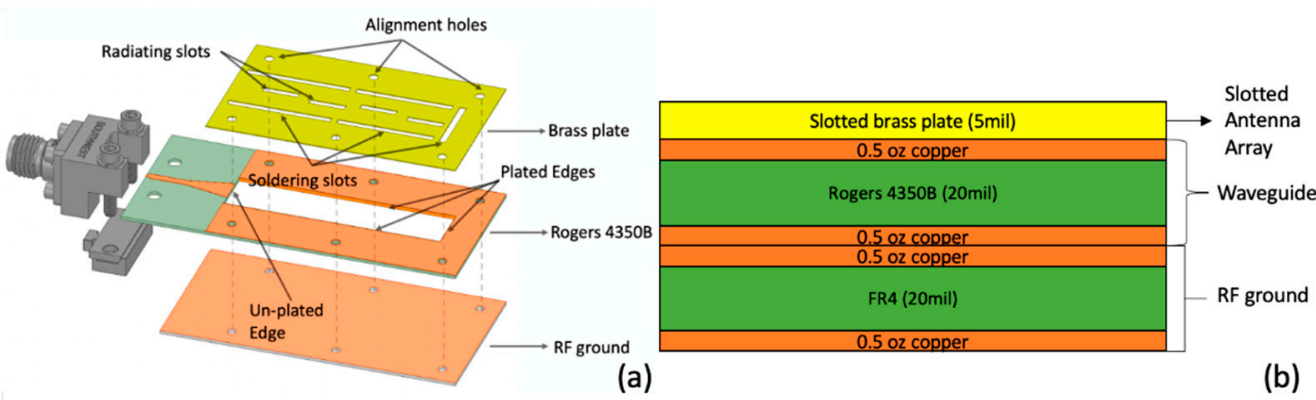

Figure 1. (a) Exploded view of air-substrate-integrated waveguide (ASIW) slot array; (b) printed circuit board (PCB) laminated structure of ASIW slot array.

The top layer is a 5 -mil brass plate. Its conductivity $(\sigma)$ is only $28 \%$ that of copper. Despite its lower conductivity, brass is much less prone to oxidation and the increased amount of zinc can provide the material with improved strength, which is mechanically more stable than most 5-mil substrates. Additionally, for waveguide structures with a large conductor area, simulation results show that a brass-based waveguide only has $4 \%$ higher attenuation at $30 \mathrm{GHz}$ than the copper counterpart (Figure 2).

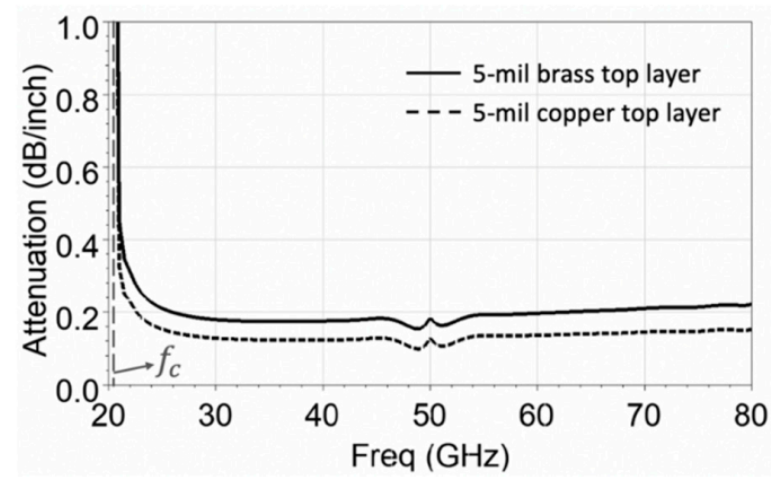

Figure 2. Attenuation of ASIW with a 5-mil brass top layer $\left(\sigma=1.5 \times 10^{7} \mathrm{~S} / \mathrm{m}\right)$ and a 5-mil copper top layer $\left(\sigma=5.8 \times 10^{7} \mathrm{~S} / \mathrm{m}\right)$. The root mean square (RMS) surface roughness is set to 1 for both materials.

In our design, the brass-plate has four radiating slots in the middle, six alignment holes on the sides to ensure accurate placement, and five additional slots around the waveguide for soldering. These additional slots are only used for prototyping, which can be eliminated in the actual manufacturing process with proper conductive bonding or mechanical assembly. The purpose of soldering slots surrounding the waveguide instead of using screws is to improve the prototyping accuracy. Although the 5-mil brass plate is stronger than copper and most 5-mil substrates, this thin layer is still susceptible to bending. There can be small air gaps between adjacent screws that can cause wave 
leakage and reduce antenna efficiency. The middle layer includes a microstrip line (MSL) and a hollow waveguide, which are fabricated on a 20-mil Rogers RO4350B substrate (Rogers Corporation, Chandler, Arizona). RO4350B has a relative permittivity $\left(\varepsilon_{r}\right)$ of 3.66 and a dielectric loss tangent $(\tan \delta)$ of 0.0037 , which provide a good performance at high frequencies with a relatively low cost. The MSL is gradually tapered to allow the quasi-TEM mode to smoothly transition into $\mathrm{TE}_{10}$ mode of the waveguide. To avoid short-circuiting the input of the waveguide, only three edges of the waveguide are copper plated as shown in Figure 1a. The top layer of the bottom substrate serves as the ground, and thus other lowcost material may be chosen. Since the waveguide shares the same physical structure as the hollow rectangular waveguide, the width $a$ of the waveguide can be simply determined by

$$
a=\frac{c}{2 f_{c}}
$$

In which $c$ is the speed of light in free space and $f_{c}$ is the $\mathrm{TE}_{10}$ mode cutoff frequency, which is $20.27 \mathrm{GHz}$ in this design. The detailed dimensions of the design are shown in Figure 3.

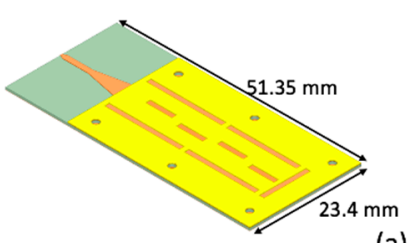

(a)

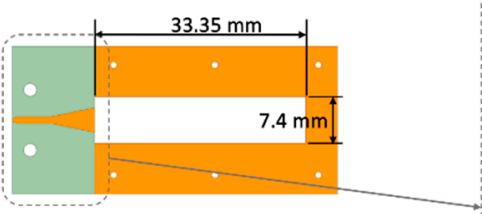

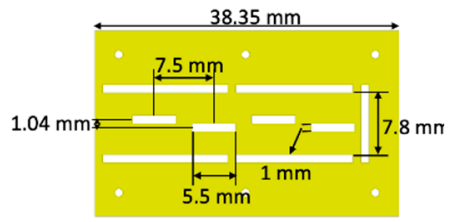

(b)

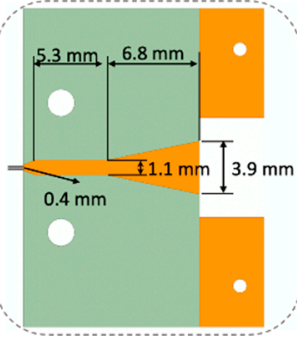

(c)

Figure 3. Dimensions of the $28 \mathrm{GHz}$ ASIW slot array. (a) Overall size (b) Dimensions of the 5-mil top slotted brass plate (c) Dimensions of the waveguide and microstrip line (MSL)-waveguide transition.

\section{Antenna Array Fabrication}

The individually milled layers and the assembled prototype are shown in Figure 4.
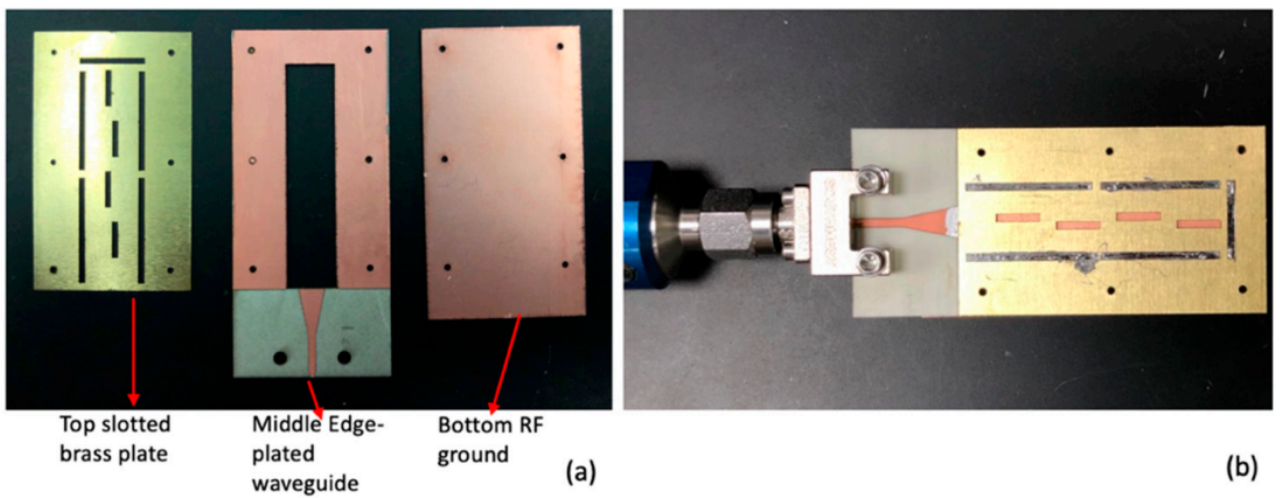

Figure 4. (a) Fabricated individual layers before assembly; (b) assembled prototype of proposed ASIW slot array under test.

For prototyping, the radiating slots on the brass-plate, the waveguide, and microstrip lines were cut and milled using LPKF Proto Laser S4 (LPKF Laser and Electronics, Garbsen, Germany). After cutting, the edges of the waveguide were copper-plated using an LPKF 
Contac S4 plating machine (LPKF Laser and Electronics, Garbsen, Germany). To plate only three edges of the waveguide, the RO4350B substrate was cut to form a five-sided hollow polygon as shown in Figure 5.

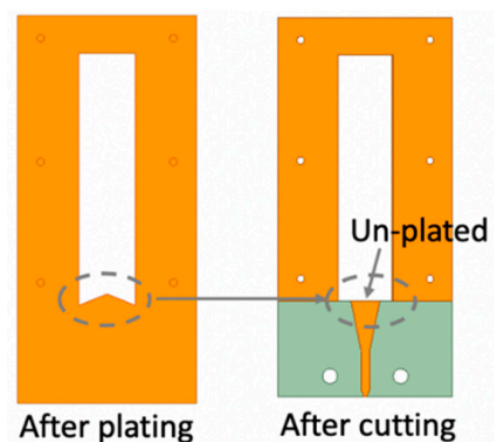

Figure 5. Three-edged copper plating process. The small triangle is cut after copper plating, such that the MSL-waveguide transition is not short-circuited.

After copper-plating, the protruding small triangle was cut out so that the input edge of the waveguide was not covered with copper. For the prototype, all the layers were aligned and soldered manually in the lab. Additionally, the surface roughness of the in-house plated copper layer could be much higher than the specification. These fabrication inaccuracies can be reduced with the standard PCB manufacturing process. To characterize the antenna efficiency more accurately, the effect of surface roughness will be discussed in the next section.

\section{Results and Discussion}

\subsection{Simulated and Measured Antenna Performance}

The ASIW slot array is simulated and analyzed in ANSYS Electronics Desktop (Canonsburg, PA, USA). The fabricated antenna is characterized in our anechoic chamber. As shown in Figure 6, the electric field of $\mathrm{TE}_{10}$ mode is uniformly distributed inside the ASIW with radiating slots.

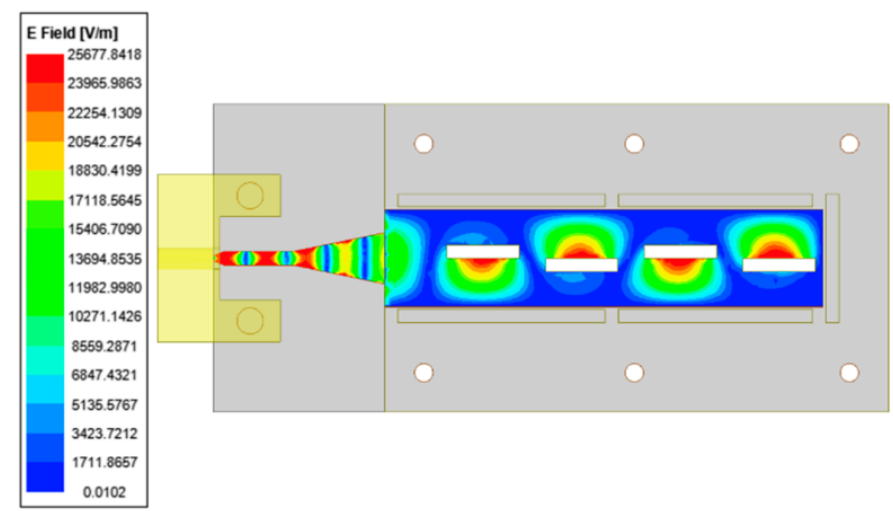

Figure 6. Electric-field distribution on the MSL and inside the ASIW with radiating slots at $28 \mathrm{GHz}$.

The measured and simulated reflection coefficients and realized gain are shown in Figure 7. In the simulation, the surface roughness of copper and brass layers is considered. The fabricated array has an impedance bandwidth (where $\left|S_{11}\right| \leq-10 \mathrm{~dB}$ ) of $10.3 \%$ (26.7-29.6 GHz). Within this bandwidth, the measured realized gain is 7.6-10.1 dBi, which is slightly less than the simulation results especially at higher frequencies. This difference is mainly caused by the non-perfect surface roughness model, which will be discussed in Section 4.2. 


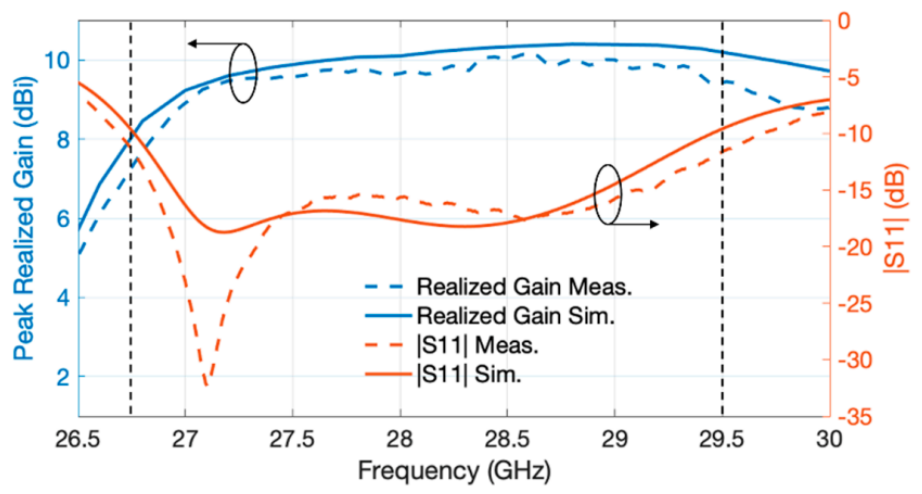

Figure 7. Simulated and measured peak realized gain and reflection coefficient of ASIW slot array.

In Figure 8, the measured and simulated radiation patterns at $27 \mathrm{GHz}, 28 \mathrm{GHz}$, and $29.5 \mathrm{GHz}$ are normalized and plotted. Both E-plane and H-plane radiation patterns agree well with the simulation results. Besides, the slot array maintains a centered main beam from $27 \mathrm{GHz}$ to $29.6 \mathrm{GHz}$ with a maximum of $3^{\circ}$ tilting at $27 \mathrm{GHz}$. This ensures a wide range of frequency scanning required by modern wireless communications.
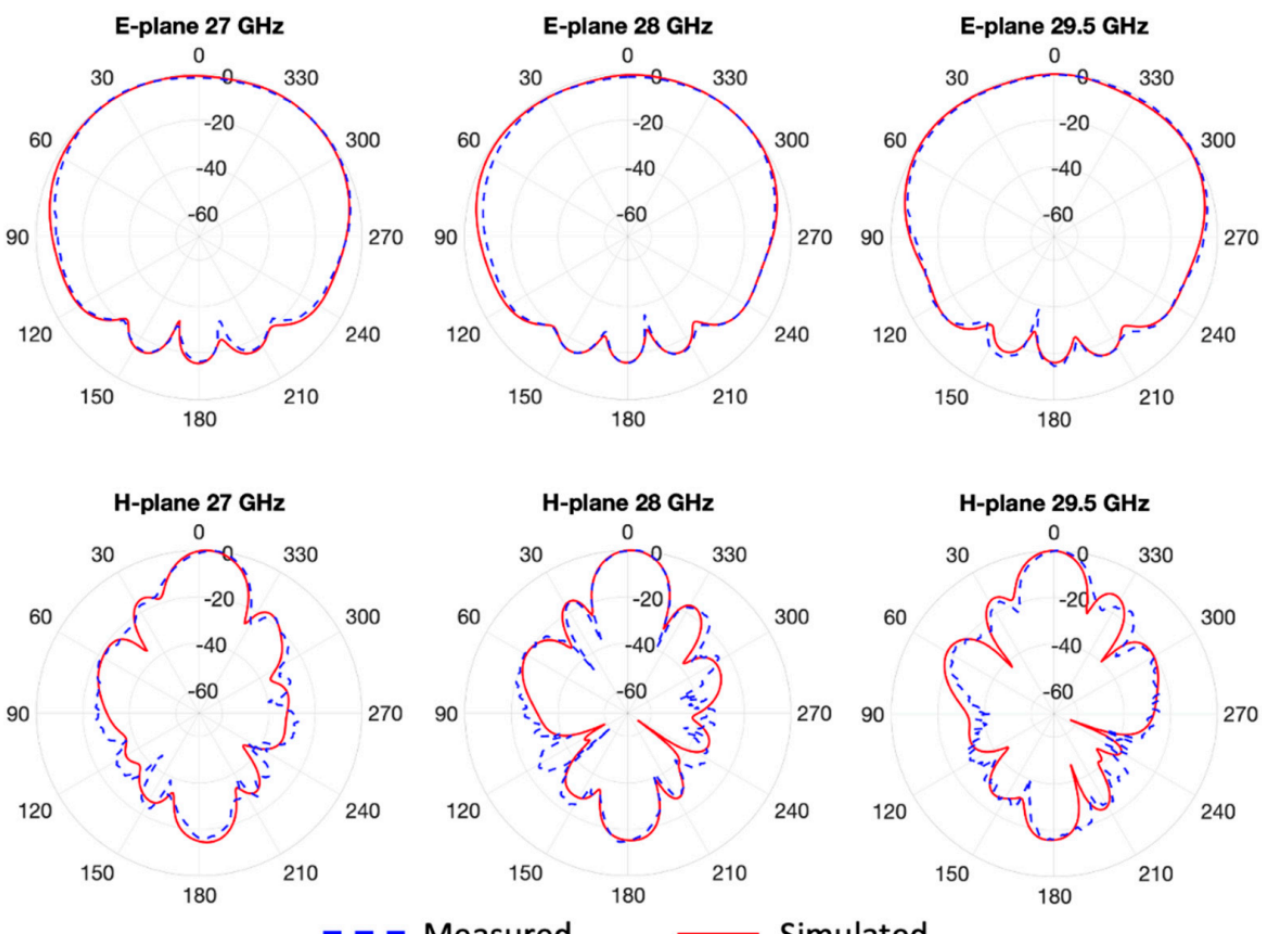

Figure 8. Simulated and measured radiation patterns (E-plane and H-plane) of ASIW slot array at $27 \mathrm{GHz}, 28 \mathrm{GHz}$, and $29.5 \mathrm{GHz}$.

For the efficiency calculation of both measured and simulated array, the reference plane is set to the input of the end-launch connector. Thus, return loss caused by impedance mismatch, as well as surface roughness, dielectric, and conductor loss of the MSL section, is considered. When the MSL section is de-embedded and the reference plane is set to the input of waveguide, simulated antenna efficiency is as high as $98 \%$. However, to evaluate the antenna performance when integrating with planar circuits, the effect of the MSL section has to be considered. By taking the ratio of total radiated power over incident power, the measured efficiency is $84 \%$ at $28.6 \mathrm{GHz}$, which is $4 \%$ lower than the simulated efficiency of $88 \%$ with the consideration of surface roughness $\left(R_{R M S}\right)$. This reduction is mainly caused by two factors. One is the fabrication error mentioned in Section 3, the 
other is the limitation of surface roughness model. When $R_{R M S}=0$, the simulated total efficiency is $92 \%$ as shown in Figure 9, causing a larger discrepancy between simulated and measured results.

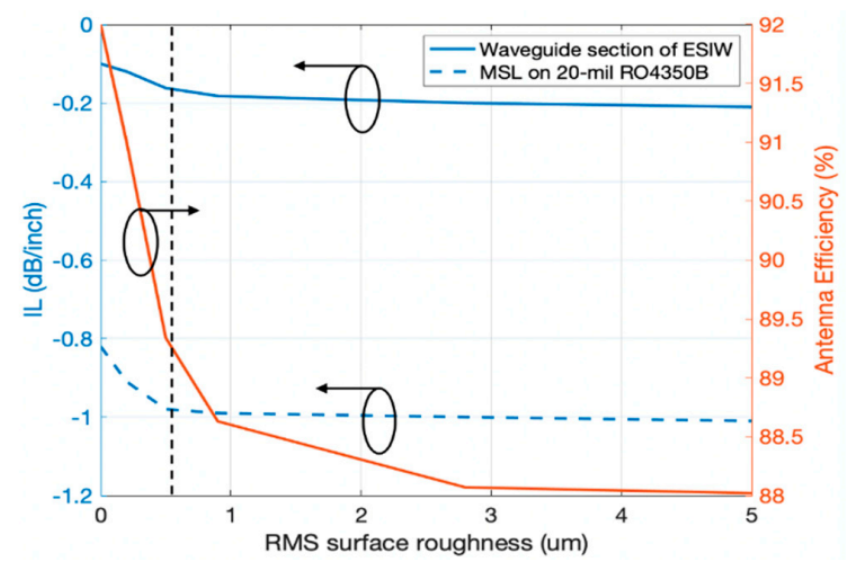

Figure 9. Simulated insertion loss (IL) and antenna efficiency versus RMS surface roughness at $28 \mathrm{GHz}$ using Groisse's surface roughness model.

\subsection{Surface Roughness Model}

The surface roughness model used in the simulation is based on Groisse et al. [9], who described a factor $C_{S}$ for correcting power loss $(P L)$ caused by $R_{R M S}$

$$
\begin{gathered}
P L_{\text {rough }}=P L_{\text {smooth }} \times C_{S}, \\
C_{S}=1+\exp \left\{-\left[\frac{\delta}{2 R_{R M S}}\right]^{1.6}\right\},
\end{gathered}
$$

In the equations, $P L_{\text {rough }}$ is the power loss through a rough conductor, $P L_{\text {smooth }}$ is the power loss through a smooth conductor, and $\delta$ is the skin depth. This correction factor works well for conductors with moderate surface roughness. As $R_{R M S}$ and frequency increases, $C_{S}$ will saturate at two. This also means the maximum power loss caused by $R_{R M S}$ is only twice the smooth conductor.

To evaluate the surface roughness effect on the ASIW slot array performance using Groisse's model, the antenna efficiency and insertion loss (IL) of MSL and air-filled waveguide versus $R_{R M S}$ are plotted in Figure 9.

Because the waveguide is filled with air, the loss is mainly caused by the MSL section. When $R_{R M S}$ is lower than $0.6 \mu \mathrm{m}$, the IL increases; antenna efficiency decreases linearly with higher $R_{R M S}$, while they start to saturate when $R_{R M S}$ is higher than $1 \mu \mathrm{m}$. However, in real measurement, this saturation will not occur and the insertion loss for rough conductors at high frequencies can be substantially higher than the calculated one [10]. For our array prototype, a low-cost RO4350B with electrodeposited (ED) copper was used, which gave us an $R_{R M S}$ of $2.8 \mu \mathrm{m}$. The in-house copper-plating process could have also produced a higher $R_{R M S}$. These could have been the main causes of the lower measured efficiency and were not predicted by Groisse's model. This result also confirms that the ASIW slot array fabricated on a low-cost substrate material can have improved efficiency with better surface treatment, such as reversed treated ED copper $\left(R_{R M S}=9 \mu \mathrm{m}\right)$ and standard copper plating techniques.

\subsection{Antenna Performance Comparison}

With the existence of the well-developed SIW slot array, the purpose of the proposed ASIW slot array is to produce better antenna efficiency with a lower budget. For comparison, a SIW slot array using the same 20-mil Rogers RO4350 (Rogers Corporation, Chandler, Arizona) was designed. Both arrays were optimized to the similar operating frequencies, and their reflection coefficients and efficiencies are shown in Figure 10a. 

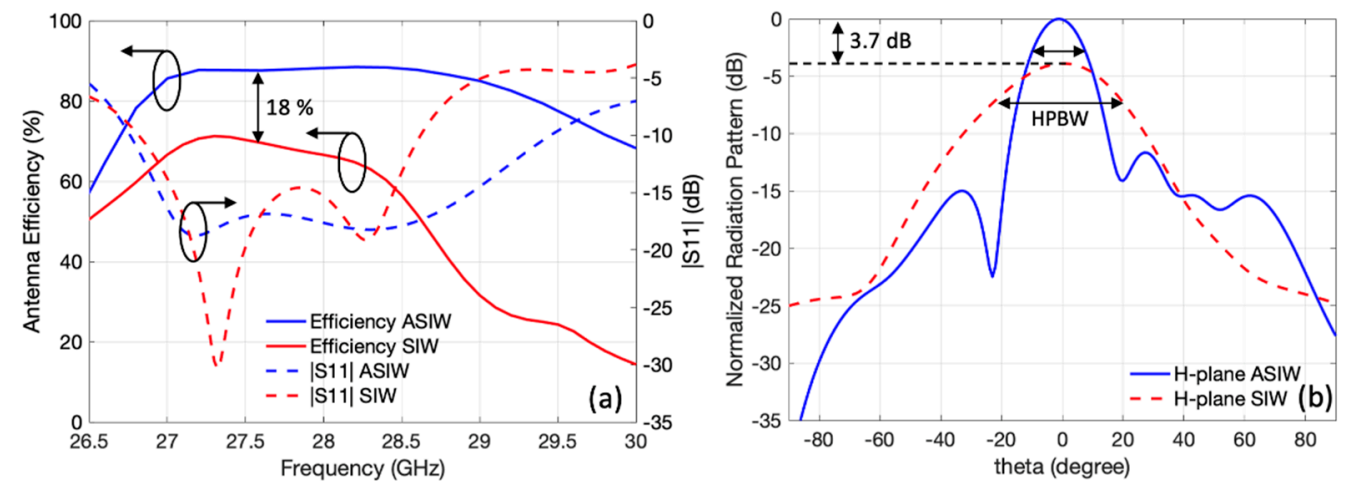

Figure 10. Comparison between ASIW slot array and substrate-integrated waveguide (SIW) slot array optimized to the similar frequency range using the same 20-mil Rogers RO4350B (Rogers Corporation, Chandler, Arizona) substrate. (a) Total antenna efficiency and IS11 I of ASIW slot array and SIW slot array. (b) Peak realized gain and half-power beamwidth (HPBW) of ASIW slot array and SIW slot array.

The SIW slot array has 30\% less bandwidth than the ASIW slot array. Although they have a similar level of reflection coefficient at $27.6 \mathrm{GHz}$, the efficiency of the ASIW slot array is $18 \%$ higher than that of the SIW slot array. Furthermore, the hollow waveguide structure of ASIW can provide a much larger aperture size than the dielectric-filled SIW, as shown in Figure 11.
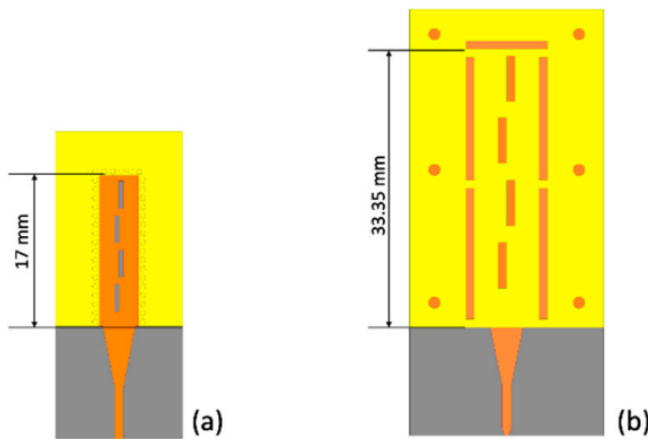

Figure 11. Size comparison between SIW slot array and ASIW slot array optimized to the similar frequency range using the same 20-mil RO4350B material. (a) SIW slot array; (b) ASIW slot array.

This larger aperture of the $1 \times 4$ SIW slot array provides a $3.7 \mathrm{~dB}$ higher gain and a $58 \%$ narrower H-plane half-power beamwidth (HPBW) than the $1 \times 4$ SIW slot array as shown in Figure 10b. These can be beneficial for some mm-wave applications. To achieve the same gain and beamwidth, the SIW slot array would have to have a longer dielectric-filled waveguide and more radiating slots. This would result in even higher dielectric loss within the waveguide.

The performance of ASIW slot array is also compared to other works, as shown in Table 1. As discussed before, the realized gain and total efficiency of the ASIW array not only include the impedance mismatch and connector loss but also dielectric, conductor, and surface roughness loss of the MSL feeding. When these effects are de-embedded by setting the reference plane to the input of the waveguide as described in Parment et al. [8], the simulated antenna efficiency of ASIW slot array is $98 \%$ and the realized gain is $11.3 \mathrm{dBi}$ at $29.5 \mathrm{GHz}$. Compared to AFSIW, the advantage of proposed ASIW is more pronounced at higher mmWave frequencies, since the densely placed vias of AFSIW are replaced with metallic walls, which can be more accurately fabricated. The ESIW slot array presented in Qi et al. [11] does not suffer from the loss of planar transition, because the waveguide is directly fed by a $2.4 \mathrm{~mm}$ connector, which makes it incompatible with planar circuit fabrication technologies. Overall, the proposed ASIW slot array can achieve a high 
efficiency with a wide bandwidth. Meanwhile, the array can still be integrated with other planar circuits using the MSL to waveguide transition.

Table 1. Comparison between different SIW slot array technologies.

\begin{tabular}{|c|c|c|c|c|c|c|}
\hline $\begin{array}{l}\text { Slot Array } \\
\text { Type }\end{array}$ & Realized Gain (dBi) & $\begin{array}{l}\text { Number of } \\
\text { Elements }\end{array}$ & $\begin{array}{l}\text { Center } \\
\text { Frequency } \\
\text { (GHz) }\end{array}$ & Efficiency & $\begin{array}{l}\text { Fractional Bandwidth } \\
\quad\left(\left|\mathbf{S}_{11}\right| \leq-10 \mathrm{~dB}\right)\end{array}$ & $\begin{array}{l}\text { Compatibility with } \\
\text { Planar Circuits }\end{array}$ \\
\hline SIW [2] & 15.7 & $4 \times 4$ & 10.0 & n.a. & $6 \%$ & Yes \\
\hline SIW [12] & 22.8 & $4 \times 32$ & 24.0 & $67 \%$ & $1.7 \%$ & Yes \\
\hline AFSIW [8] & $11.5^{1}$ & $1 \times 4$ & 30.5 & $97.4^{1}{ }^{1,2}$ & $8.7 \%$ & Yes \\
\hline ESIW with Cavity Loading [11] & 15.5 & $1 \times 6$ & 38.5 & $90.7 \%$ & $12.7 \%$ & No \\
\hline ESIW [13] & 15 & $1 \times 10$ & 16.25 & $90.78 \%$ & $4.3 \%$ & Yes \\
\hline ASIW (this work) & $10.1\left(11.3^{1,2}\right)$ & $1 \times 4$ & 28.15 & $84 \%^{3}\left(98 \%{ }^{1,2}\right)$ & $10.7 \%$ & Yes \\
\hline
\end{tabular}

${ }^{1}$ Reference plane is set to the input of waveguide, transition section is de-embedded; ${ }^{2}$ simulated; ${ }^{3}$ total efficiency: return loss included.

\section{Conclusions}

A high-efficiency and low-loss ASIW slot array design is proposed in this paper. The hollow waveguide implementation with a simple tapered MSL feeding eliminates the substrate loss and wave-leakage loss existing in the conventional SIW slot arrays. The proposed four-element array covering the $28 \mathrm{GHz} 5 \mathrm{G}$ band shows $18 \%$ higher efficiency than the SIW slot array designed on the same substrate. During the analysis, surface roughness has a major contribution to the loss of the ASIW slot array. With low-roughness surface treatment, higher antenna efficiency can be achieved with low-cost substrate materials.

Author Contributions: Conceptualization, J.-B.Y. and L.L.; methodology, L.L. and J.-B.Y.; software, L.L. and J.-B.Y.; validation, L.L.; formal analysis, L.L.; investigation, L.L. and J.-B.Y.; resources, L.L. and J.-B.Y.; data curation, L.L.; writing—original draft preparation, L.L.; writing-review and editing, L.L. and J.-B.Y.; visualization, L.L.; supervision, J.-B.Y.; project administration, J.-B.Y.; funding acquisition, J.-B.Y. All authors have read and agreed to the published version of the manuscript'

Funding: This research was funded by The University Corporation for Atmospheric Research (UCAR): National Oceanic and Atmospheric Administration (NOAA), grant number 26548 and Aerotenna, Inc. grant number 25929.

Institutional Review Board Statement: Not applicable.

Informed Consent Statement: Not applicable.

Data Availability Statement: The data presented in this study are available on request from the corresponding author.

Conflicts of Interest: The authors declare no conflict of interest.

\section{References}

1. Deslandes, D.; Wu, K. Integrated microstrip and rectangular waveguide in planar form. IEEE Microw. Wirel. Compon. Lett. 2001, 11, 68-70. [CrossRef]

2. Yan, L.; Hong, W.; Hua, G.; Chen, J.; Wu, K.; Cui, T.J. Simulation and experiment on SIW slot array antennas. IEEE Microw. Wirel. Compon. Lett. 2004, 14, 446-448. [CrossRef]

3. Ding, Y.; Wu, K. A $4 \times 4$ Ridge Substrate Integrated Waveguide (RSIW) Slot Array Antenna. IEEE Antennas Wirel. Propag. Lett. 2009, 8, 561-564. [CrossRef]

4. Navarro, D.V.; Carrera, L.F.; Baquero, M. A SIW slot array antenna in Ku band. In Proceedings of the Fourth European Conference on Antennas and Propagation, Barcelona, Spain, 12-16 April 2010; pp. 1-4.

5. Xu, J.; Chen, Z.N.; Qing, X.; Hong, W. 140-GHz TE20-Mode Dielectric-Loaded SIW Slot Antenna Array in LTCC. IEEE Trans. Antennas Propag. 2012, 61, 1784-1793. [CrossRef]

6. Ranjkesh, N.; Shahabadi, M. Reduction of dielectric losses in substrate integrated waveguide. Electron. Lett. 2006, 42, 1230-1231. [CrossRef]

7. Parment, F.; Ghiotto, A.; Vuong, T.-P.; Duchamp, J.-M.; Wu, K. Air-Filled Substrate Integrated Waveguide for Low-Loss and High Power-Handling Millimeter-Wave Substrate Integrated Circuits. IEEE Trans. Microw. Theory Tech. 2015, 63, 1228-1238. [CrossRef]

8. Parment, F.; Ghiotto, A.; Vuong, T.-P.; Duchamp, J.-M.; Wu, K. Millimetre-wave air-filled substrate integrated waveguide slot array antenna. Electron. Lett. 2015, 53, 704-706. [CrossRef] 
9. Groiss, S.; Bardi, I.; Biro, O.; Preis, K.; Richter, K. Parameters of lossy cavity resonators calculated by the finite element method. IEEE Trans. Magn. 1996, 32, 894-897. [CrossRef]

10. Horn, A.F.; Reynolds, J.W.; LaFrance, P.A. Sonnet Software: Effect of Conductor Profile on the Insertion Loss, Phase Constant, and Dispersion in Thin High Frequency Transmission Lines. Available online: https://www.sonnetsoftware.com/support/ downloads/publications/5-TA1_Al_Horn.pdf (accessed on 9 January 2020).

11. Qi, Z.; Li, X.; Xiao, J.; Zhu, H. Low-Cost Empty Substrate Integrated Waveguide Slot Arrays for Millimeter-Wave Applications. IEEE Antennas Wirel. Propag. Lett. 2019, 18, 1021-1025. [CrossRef]

12. Xu, J.; Chen, Z.N.; Qing, X. CPW Center-Fed Single-Layer SIW Slot Antenna Array for Automotive Radars. IEEE Trans. Antennas Propag. 2014, 62, 4528-4536. [CrossRef]

13. Miralles, E.; Belenguer, A.; Matéo, J.; Torres, A.; Esteban, H.; Borja, A.L.; Boria, V.E. Slotted ESIW antenna with high efficiency for a MIMO radar sensor. Radio Sci. 2018, 53, 605-610. [CrossRef] 